\title{
An online dietary and lifestyle education intervention to improve sleep quality
}

\author{
L. Lodererova ${ }^{1}$ and D. Bhakta ${ }^{1}$ \\ ${ }^{1}$ School of Human Sciences, London Metropolitan University, London, UK
}

Nutrition and sleep are important modifiable lifestyle factors for the health and well-being of the individual. Recent research suggests that there is a direction relationship between diet and sleep ${ }^{(1)}$ and sleep quality and risk of chronic diseases such as obesity, cardiovascular disease and Type 2 diabetes $^{(2)}$. Specific nutrients have been associated with promoting or obstructing sleep quality ${ }^{(3)}$. Tryptophan (TRP) has been researched and proposed to positively influence sleep quality because of its metabolic pathway to create melatonin. In the U.K, almost three quarters (74\%) of adults sleep less than seven hours a night, which is under the optimal sleep recommendations ${ }^{(4)}$. We are not aware of any studies exploring a combination of online educational dietary intake intervention focused on TRP and its effect on sleep quality. Furthermore, because of current government restrictions for COVID-19, the intervention was delivered online.

Volunteers were recruited via Facebook and Instagram social media. Dietary intake was measured using FFQ-EPIC Norfolk, and sleep quality was measured using the Pittsburgh Sleep Quality Index (PSQI). A questionnaire was used to collect information on demographics and self-reported anthropometric data. The intervention comprised of a 5-minute PowerPoint presentation video on the relationship between nutrition, lifestyle and sleep. Nutrition education was based on the Eatwell Guide, emphasising encouraging intake of foods high in tryptophan intake and decreasing alcohol and caffeine intake. Additionally, the participant received information on basic lifestyle tips on how to incorporate sleep hygiene. The FFQ-EPIC and PSQI were re-administered one month after the educational intervention. The FFQ was analysed using Nutritics to obtain estimated habitual daily dietary intakes. A paired sample t-test (SPSS v. 24) was used to assess changes before and after the intervention.

The participants $(\mathrm{n}=23)$ were adults aged between $18-25$ years and the mean body mass index (BMI) was $22.5 \mathrm{~kg} / \mathrm{m} 2$. Sleep quality improved after intervention from PSQI $=6$ to PSQI score $=5$ but did not reach significance $(p=0.06)$. Energy and nutrient intake did not significantly change before and after the intervention. Mean TRP intake, alcohol intake or caffeine intake did not significantly change. However, the study observed changes in individual subjective sleep quality in components such as improved sleep efficiency, longer sleep duration and less daytime dysfunction.

This study has shown the potential novelty of low-cost, non-invasive educational approaches with a low burden on participants to improve sleep quality via dietary advice in adults.

\section{References}

1. St-Onge MP, Mikic A \& Pietrolungo CE (2016) Adv Nutr 7, 938-949.

2. CDC (2018) Sleep and Chronic Disease Prev Chronic Dis.

3. Peuhkuri K, Sihvola N \& Korpela R (2012) Nutr Res 32, 309-319.

4. The Sleep Council (2017) The Great British Bedtime Report. 